\title{
SAR I mage De-noising Based on Residual Image Fusion and Sparse Representation
}

\author{
Xiaole $\mathrm{Ma}^{1}$, Shaohai $\mathrm{Hu}^{1 *}$ and Dongsheng Yang ${ }^{1}$ \\ ${ }^{1}$ Institute of Information Science, Beijing Jiaotong University \\ Beijing, 100044 - China \\ [e-mail: shhu@bjtu.edu.cn] \\ *Corresponding author: Shaohai Hu
}

Received May 30, 2018; revised September 11, 2018; revised October 7, 2018; accepted December 7, 2018; published July 31, 2019

\begin{abstract}
Since the birth of Synthetic Aperture Radar (SAR), it has been widely used in the military field and so on. However, the existence of speckle noise makes a good deal inconvenience for the subsequent image processing. The continuous development of sparse representation (SR) opens a new field for the speckle suppressing of SAR image. Although the SR de-noising may be effective, the over-smooth phenomenon still has bad influence on the integrity of the image information. In this paper, one novel SAR image de-noising method based on residual image fusion and sparse representation is proposed. Firstly we can get the similar block groups by the non-local similar block matching method (NLS-BM). Then SR de-noising based on the adaptive K-means singular value decomposition (K-SVD) is adopted to obtain the initial de-noised image and residual image. The residual image is processed by Shearlet transform (ST), and the corresponding de-noising methods are applied on it. Finally, in ST domain the low-frequency and high-frequency components of the initial de-noised and residual image are fused respectively by relevant fusion rules. The final de-noised image can be recovered by inverse ST. Experimental results show the proposed method can not only suppress the speckle effectively, but also save more details and other useful information of the original SAR image, which could provide more authentic and credible records for the follow-up image processing.
\end{abstract}

Keywords: Sparse representation, residual image, Shearlet transform, image fusion, SAR image de-noising 


\section{Introduction}

As one of microwave imaging radars with all-time and all-weather, SAR can produce radar images with high resolution, which has more abundant information compared with optical images. Thus, it has been widely used in special technology, strategic and tactical in military field, etc. Nevertheless, the speckle noise caused by its coherent imaging mechanism, has a serious impact on the follow-up work like image enhancement and recovery. Thus, how to suppress even eliminate the speckle noise effectively, has become one of the main tasks in the SAR image study [1].

Until now, a great deal of SAR image de-noising methods have been proposed, which can be divided into three types: image de-noising based on spatial domain, image de-noising based on transform domain and image de-noising based on SR [2]. For spatial domain methods, they mainly use the similarity between the pixels of the image. While transform domain methods usually adopt some orthogonal basis functions to represent the noisy image and obtain different frequency sub-band coefficients; the image de-noising can be realized by processing those coefficients. The common multi-scale geometric transforms [1] which are widely used in the speckle suppressing are dual tree complex wavelet transform (DTCWT), Contourlet, Shearlet etc. Since the image contains many different kinds of characteristics to express information, if we only use the fixed orthogonal basis function, which can hardly represent all the characteristics of the image, the noise and real information cannot be completely separated [3].

With the research and continuous development of multidimensional signal sparse representation, the signal representation theory of complete and over-complete sparse decomposition have attracted tremendous attention and achieved great success in SAR image field [4]. For example, Chenglong Wang [5] proposed SAR image despeckling based on the nonlocally centralized sparse representation model, and Xiao Quan [6] proposed image denoising based on adaptive over-complete sparse representation. Compared with the image de-noising based on transform domain, the SR one can also be realized by mapping the noisy image into other domains. Differently, SR does not use the fixed basis function to represent the image, while it can sparsely represent the noisy image with the redundant dictionary, including K-SVD [7] and so on. Since the noise is not the sparse component, we can realize the image de-noising by this representation, which can depart the signal and noise clearly.

In the SR field of the image, generating a dictionary is an important precondition and a crucial link for representing the image signal sparsely. Although the over-complete dictionary has good effect for the image de-noising, it usually takes much time during the dictionary training so that the instantaneity of the algorithm is seriously influenced. The adaptive dictionary training is simple and fast; however, because of the limitations of the image itself, in most cases, the generated dictionary cannot availably represent some characteristics in the original image, which leads to the losing of some essential detail information and over-smoothing phenomenon, finally causing the decline of the de-noising effect. To overcome these aforementioned shortcomings, in this paper, we first extract the useful information from the residual image, which is obtained by the image de-noising based on SR; then fuse them into the initial de-noised image of SR de-noising by the image fusion methods; in the end we can obtain the final de-noised image.

The rest of this paper is organized as follows. Section 2 introduces the image de-noising based on K-SVD; while in Section 3 the residual image de-noising method based on ST domain is 
discussed. The fusion of the residual image and initial de-noised image is illustrated in Section 4, and in this part the framework of the proposed method is shown, too. Experiments and its results can be seen in Section 5. At last, we make the conclusion in Section 6 and the future scope is discussed in Section 7.

\section{The Image De-noising Based on Improved K-SVD}

K-SVD is one of the image de-noising methods based on over-complete sparse decomposition $[3,8]$. Since the dictionary matrix is over-complete, there are a lot of solutions of the matrix, which means the sparse coefficients of the image is not sole. It is well known that the sparser the solution is, the better the reconstructed image is. Thus, we should choose the sparsest solutions to reconstruct the image.

Suppose the over-complete dictionary matrix is $D \in R^{M \times k}$, and the noisy is $I \in R^{M \times N}$. SR model can be simplified as Eq.(1).

$$
\hat{\alpha}=\arg \min _{\alpha}\|\alpha\|_{0} \quad \text { s.t. }\|D \alpha-I\|_{2}^{2} \leq \varepsilon
$$

where $\alpha$ denote the sparse coefficients. In most cases, $\|\alpha\|_{0} \leq L<<M$, and $L$ denotes the maximum value of the sparseness. When $\varepsilon$ is fixed, we can get the sparse coefficient $\alpha$ by solving the model in Eq.(1).

In order to solve SR model by orthogonal matching pursuit (OMP) method better, we can change the model in Eq.(1) into the following model in Eq.(2).

$$
\hat{\alpha}=\arg \min _{\alpha}\|D \alpha-I\|_{2}^{2}+\mu\|\alpha\|_{0}
$$

where $\mu$ denotes the penalty factor. As usual, we abstract the noisy image as the additive model $Y=X+n$. Among the aforementioned model, $Y$ denotes observed SAR image in reality, $X$ denotes the ideal noise-free image and $n$ denotes the noise.

Since the dictionary training can be effecitve only if the image is small, if we use the whole noisy image to train the dictionary directly, to some extent the sparseness of the image will be influenced so the sparsest representation of the image cannot be obtained [7]. More importantly, this process ignores the similar information hidden in the image [9]. In this paper, we first divide the original noisy image into different image blocks with small size; next we obtain the similar image block groups by the non-local similar block matching method [10] and process every group by K-SVD based on OMP. By this way, we can not only obtain the sparsest representation, but also improve the algorithm's accuracy and availability.

$$
\left\{\hat{D}, \hat{\alpha}_{i j}, \hat{X}\right\}=\arg \min _{D, \alpha_{i j}, X} \lambda\|X-Y\|_{2}^{2}+\sum_{i, j} \mu_{i j}\left\|\alpha_{i j}\right\|_{0}+\sum_{i, j}\left\|D \alpha_{i j}-X_{i j}\right\|_{2}^{2}
$$

where $\lambda\|X-Y\|$ denotes the approximation degree between the noisy image $Y$ and noise-free image $X$, and it can be realized by $\|X-Y\|_{2}^{2} \leq$ Const $\cdot \sigma^{2} \cdot \sigma$ denotes the standard deviation of the noise. All of the subscripts stand for the position $(i, j)$ that refers to the center pixel of the corresponding image block.

Although we can obtain the optimal sparse representation and good de-noising results by using aforementioned SR method, it is also more possible to generate over-smooth, and lead 
the loss of the details, edges and other important information, even destroy the integrity of the image information in some serious cases. As shown in Fig. 1, Fig. 1(a) and (b) are the original SAR images token by French Space Agency and the noisy image with the ENL of $L=25$. Fig. 1 (c) is the initial de-noised image by the improved K-SVD, while Fig. 1(d) is the residual image between the original image and initial image. As can be seen in Fig. 1(c), some edges are lost and the outline is fuzzy. The residual image in Fig. 1(d) contains not only much noise, but also some detail information such as the missed outlines in Fig. 1(c). Therefore, it is essential to extract the useful information in the residual image and fuse them into the initial de-noised image. And this process determines the final de-noised results and has significant impact on the subsequent image processing.

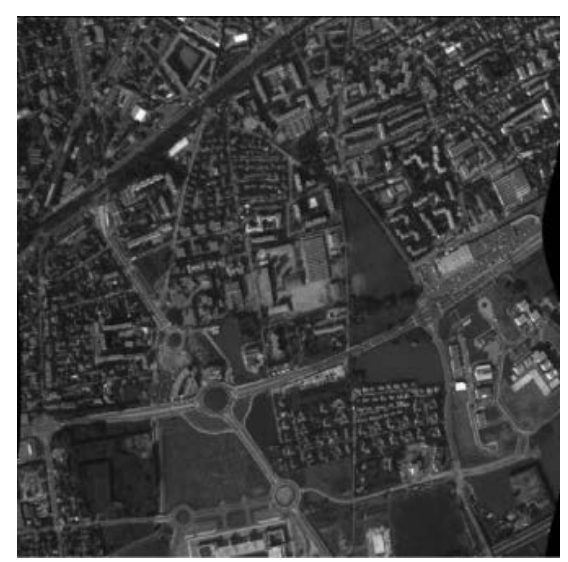

(a)

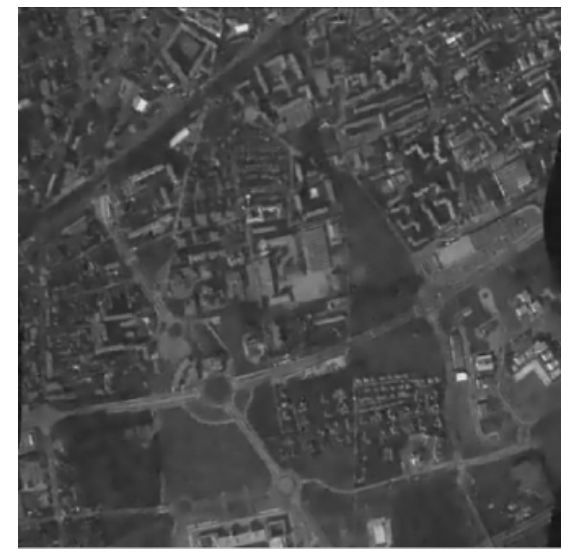

(c)

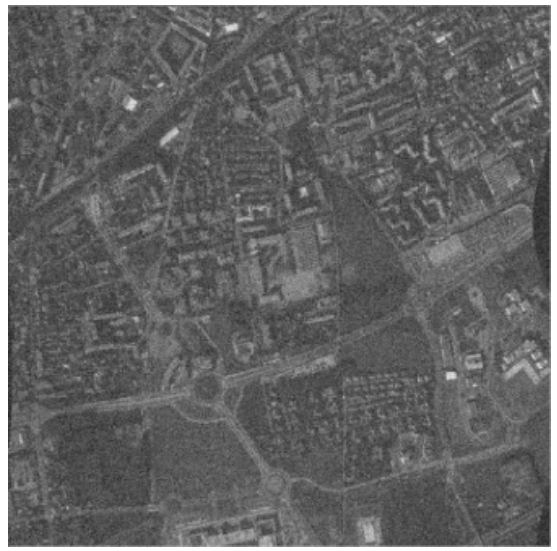

(b)

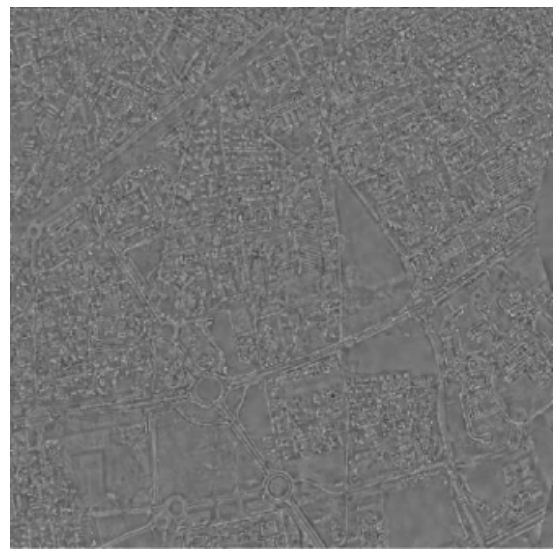

(d)

Fig. 1. The images of K-SVD (a) the original image; (b) the noisy image; (c) the initial de-noised image; (d) the residual image

\section{The Residual Image De-noising in Shearlet Domain}

As mentioned above, the de-noising methods based on SR can make the information of the original image be incomplete. In the proposed method, we need to extract the discarded useful information from the residual image. Here we introduce the residual image de-noising in 
Shearlet domain briefly and by this way, we can extract the useful detail information efficiently.

\subsection{Shearlet}

Shearlet is one of the multi-scale geometric transforms [11], which is the combination of the geometric and multi-resolution analysis by the classical theory of the affine systems. And when the dimension is two, which means $n=2$, the affine system with the synthesis of inflation can be represented as Eq.(4).

$$
\mathcal{A}_{A B}(\psi)=\left\{\psi_{j, l, k}(x)=|\operatorname{det} A| \frac{j}{2} \psi\left(B^{l} A^{j} x-k\right) ; j, l \in \mathbb{Z}, k \in \mathbb{Z}^{2}\right\}
$$

where $A=\left[\begin{array}{l}4,0 \\ 0,2\end{array}\right]$ denotes the expansion matrix with the anisotropy, and $B=\left[\begin{array}{l}1,1 \\ 0,1\end{array}\right]$ denotes the shear matrix.

The support interval of wavelet function $\psi_{j, l, k}$ in frequency domain is

$$
\sup p \widehat{\psi}_{j, l, k}^{(0)} \subset\left\{\left(\xi_{1}, \xi_{2}\right): \xi_{1} \in\left[-2^{2 j-1},-2^{2 j-4}\right] \cup\left[2^{2 j-4}, 2^{2 j-1}\right],\left|\frac{\xi_{2}}{\xi_{1}}+l 2^{-j}\right| \leq 2^{-j}\right\}
$$

As Fig. 2(a) shows, the support interval $\widehat{\psi}_{j, l, k}^{(0)}$ is a pair of trapezoidal areas, of which the size is $2^{2 j} \times 2^{j}$ and the slope is $l 2^{-j}$. Supposing $D_{0}=\left\{\left(\xi_{1}, \xi_{2}\right) \in \hat{\mathbb{R}}^{2}:\left|\xi_{1}\right| \geq \frac{1}{8},\left|\frac{\xi_{2}}{\xi_{1}}\right| \leq 1\right\}$, the function sets $\left\{\hat{\psi}^{(0)}\left(\xi A_{0}^{-j} B_{0}^{-l}\right)\right\}$ can form the decomposition figure spanned $D_{0}$ in frequency domain, which can be seen as the solid line in Fig. 2(b). In the same way, we can structure the dotted line of Fig. 2(b), which is $D_{1}=\left\{\left(\xi_{1}, \xi_{2}\right) \in \hat{R}^{2}:\left|\xi_{2}\right| \geq \frac{1}{8},\left|\frac{\xi_{1}}{\xi_{2}}\right| \leq 1\right\}$.

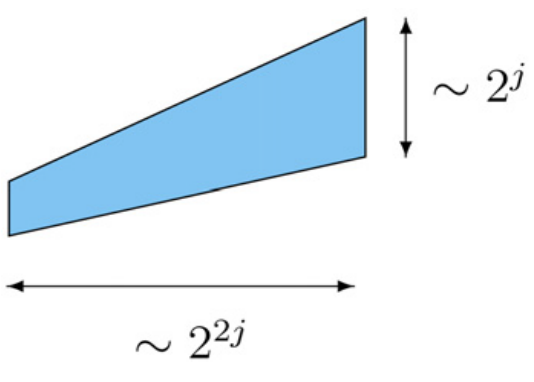

(a)

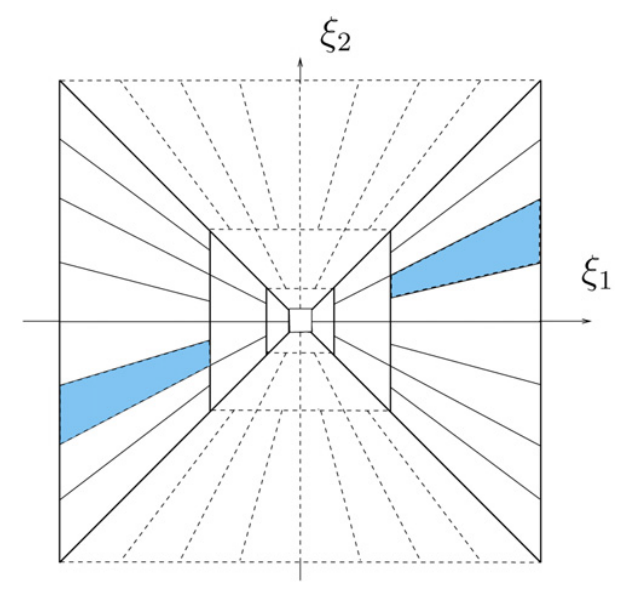

(b)

Fig. 2. Shearlet (a) the support interval of $\psi_{j, l, k}$; (b) the decomposition figure in ST domain 
Therefore, the continuous ST transform of $f$ can be defined as Eq.(6).

$$
S T_{\psi}=\left\langle f, \psi_{j, l, k}^{(d)}\right\rangle
$$

where $j \geq 0,-2^{j} \leq l \leq 2^{j}-1, k \in \mathbb{Z}^{2}, d=0,1$.

\subsection{The Residual Image De-noising in ST Domain}

After we obtain the high-frequency coefficients $C_{H}$ and low-frequency coefficients $C_{L}$ of the residual image, it is very important that adopting which kinds of image de-noising methods to processing these coefficients. Compared with usual noisy images, the residual image has much more noise. Besides, the useful information of the residual image mainly exists in the high-frequency components. So, in this paper we adopt the traditional soft threshold [12] to process the low-frequency coefficients and use the adaptive threshold to deal with the high-frequency coefficients. The soft threshold can be realized by Eq.(7).

$$
y(x)=\left\{\begin{array}{l}
\operatorname{sgn}(x)(|x|-T), \text { if }|x| \geq T \\
0, \text { otherwise }
\end{array}\right.
$$

where $T$ denotes the threshold, and sgn(.) denotes the sign function.

Since the noise and noise-free image is independent of each other [1], the model of the residual image in the high-frequency components can be simplified as Eq.(8).

$$
\sigma_{H}^{2}=\sigma_{F}^{2}+\sigma_{N}^{2}
$$

where $\sigma_{H}^{2}$ denotes the noise variance of the high-frequency components, $\sigma_{F}^{2}$ denotes the variance of the high-frequency components in the noise-free image and $\sigma_{N}^{2}$ denotes the noise variance. To make full use of the correlation between the pixels, at the sub-band of scale $i$ and direction $j$, we build a $8 \times 8$ pixel size sliding window centered on $H_{i, j}(p, q)$. Then we can get the variance of the center pixel by Eq.(9).

$$
\hat{\sigma}_{H, p, q}^{2}=\frac{1}{8 \times 8} \sum_{k=1}^{8} \sum_{l=1}^{8}\left|H_{i, j}(k, l)\right|^{2}
$$

Thus, the variance of high-frequency sub-bands with the size of $m \times n$ can be obtained by Eq.(10).

$$
\hat{\sigma}_{H}^{2}=\frac{1}{m \times n} \sum_{p=1}^{m} \sum_{q=1}^{n} \hat{\sigma}_{H, p, q}^{2}
$$

According to the method proposed by Dong-ming Li [13], we can obtain the variance of the noise.

$$
\hat{\sigma}_{N}=\frac{\operatorname{Median}\left(\left|H_{i, j}\right|\right)}{0.6745}
$$

Finally, we can obtain the variance of the de-noised image in the high-frequency

components by $\hat{\sigma}_{F}^{2}=\max \left(\left(\hat{\sigma}_{H}^{2}-\hat{\sigma}_{N}^{2}\right), 0.000001\right)$. When processing the high-frequency components with the adaptive threshold, the value of the threshold can be obtained by Eq.(12). 


$$
T_{i, j}\left(\hat{\sigma}_{H}^{2}\right)=\frac{\hat{\sigma}_{N}^{2}}{\hat{\sigma}_{F}^{2}}=\frac{\hat{\sigma}_{N}^{2}}{\sqrt{\max \left(\left(\hat{\sigma}_{H}^{2}-\hat{\sigma}_{N}^{2}\right), 0.000001\right)}}
$$

\section{SAR Image De-noising Based on Residual Image Fusion and Sparse Representation}

After we obtain the high-frequency and low-frequency de-noised coefficients of the initial de-noised image and residual image, how to fuse them together by the image fusion methods is another important step in the proposed algorithm. Since the residual image includes much noise and a few detail information of the original image, and both of them mainly exist in the high-frequency components; besides, the detail information in the residual image is completely complementary in the high-frequency components of the initial de-noised image. Therefore, for the high-frequency coefficients of the images, we adopt the fusion rule that adds the different coefficients with the weighting factors, which can be seen in Eq.(13).

$$
C_{H_{F}}=a C_{H}+b C_{H_{o}}
$$

where $C_{H_{F}}$ denotes the high-frequency coefficients of the fused de-noised image, $C_{H}$ denotes the high-frequency coefficients of the residual image and $C_{H_{0}}$ denotes the high-frequency coefficients of the initial de-noised image by the SR de-noising where exist most the high-frequency information of the original image. $a$ and $b$ denote the weighting factors of the corresponding coefficients respectively. To make the final de-noised image accurate and save more original image information, here we set $b=1$. According to the experiments on different images and values of the parameter $a$, we found that 1.2 is the best. As a result, we make $a=1.2, b=1$.

For the low-frequency coefficients, we adopt the traditional "choose max" fusion rule to fuse the coefficients, which can be seen in Eq.(14).

$$
C_{L_{F}}=\max \left(C_{L}, C_{L_{o}}\right)
$$

where $C_{L_{F}}, C_{L}$ and $C_{L_{o}}$ denote the low-frequency coefficients of the de-noised SAR image the residual image and the initial de-noised image respectively.

Normally, the speckle noise is fully developed [14]. Moreover, it is a kind of the multiplicative noises, which obeys the $\Gamma$ distribution and has the second order stationarity. And the mean of the speckle is one; its variance and equivalent numbers of looks are inversely proportional. Before de-noising the SAR image, we usually process the noisy image with logarithmic transform to obtain the corresponding additive noise model. And after the whole process of the image de-noising, an exponential transform is performed to obtain the final de-noised image. The flow chart of the proposed algorithm can be seen in Fig. 3.

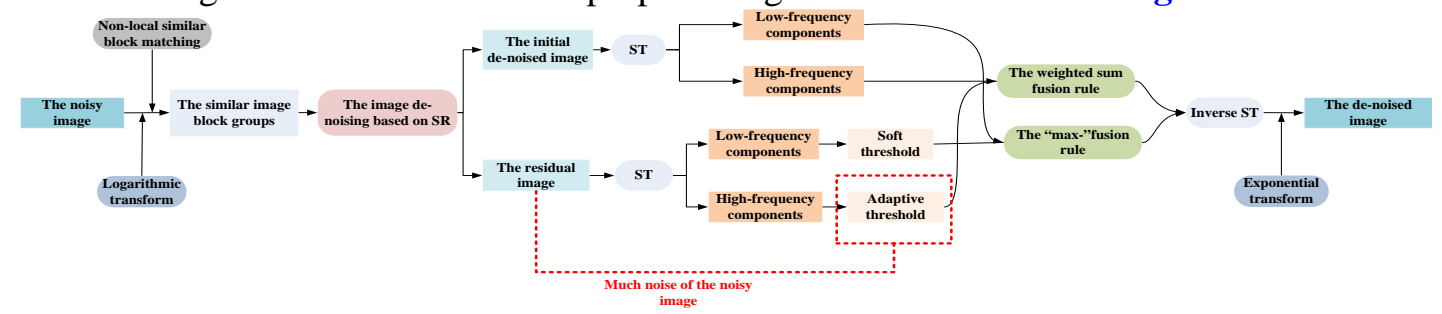

Fig. 3. The flow chart of the proposed algorithm 


\section{The Experimental Results and Analysis}

To verify the availability and reliability of the proposed algorithm, we compare our algorithm with the image de-noising based on Shearlet (ST) [1], PPB [15], SAR-BM3D [16], K-SVD [3], SR and image fusion (K-SVD+Wavelet) [17]. Moreover, all the experiments were carried out by Matlab codes on an Intel Core i5 $3.1 \mathrm{GHz}$ with $4 \mathrm{~GB}$ RAM.

At first, we take the experiments of the SAR image with the size of $512 \times 512$ took by French Space Agency. Fig. 4(a) is the original image, and we add the speckle to Fig. 4(a) with the equivalent number of looks $L=5 \sim 40$. The noisy image with $L=25$ shown in Fig. 4(b) is the most typical one among all the situations. So, we show more detail of it and discuss the results of its de-noised images by different methods.

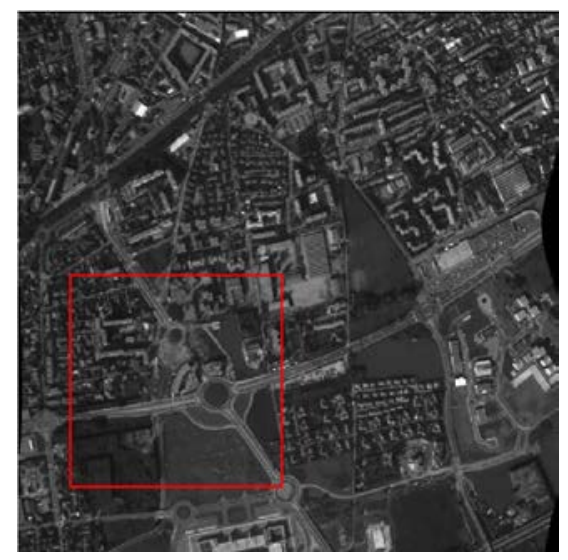

(a)

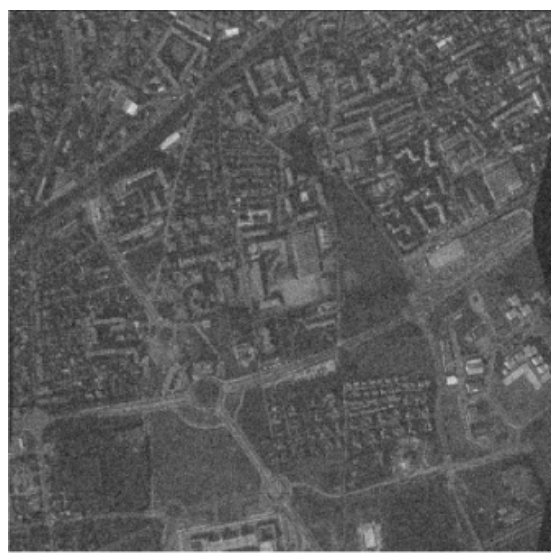

(b)

Fig. 4. The SAR image took by French Space Agency (a) the original image; (b) the noisy image

Fig. 5(a) (f) are the de-noised images by various of de-noising methods such as ST, PPB, SAR-BM3D, K-SVD, K-SVD+Wavelet and the proposed method. And Fig. 6 shows more details about the de-noised region in red rectangle of Fig. 4(a). From Fig. 5 and Fig. 6(a) we can see, the de-noised image by ST loses a lot of detail information and its edges are very blurry. Although the de-noised images by PPB and SAR-BM3D can save more edges, they involve some artificial texture in the homogeneous area, which can disturb the information of the original image. By comparing the lower right corner in Fig. 6(b) and (c), the de-noised image by PPB is better. To some extent, using K-SVD can obtain qualified de-noising result and suppress the artificial texture. However, compared with ordinary optical images, SAR image contains more detail information which can be easily ignored and cannot be represented ultimately when de-noising the image by SR, and this leads the loss of some useful information, which is shown in Fig. 5(d), seriously influencing the completeness of the original image. As shown in Fig. 5(e), compared with SR method shown in Fig. 5(d), the image de-noising based on dictionary learning and residual information fusion proposed by Dong Mingkun can reserve more detail information of the original image; but it adopts wavelet transform which cannot accurately express the directional information of the image edges. Compared with Fig. 5(e), the de-noised image by the proposed method not only has less artificial texture, but saves more useful details of the original image, especially the image edges. In addition, Fig. 5(f) and Fig. 6(f) shows the proposed method has the best visual effect among all the results and the proposed method is prior to the state of the art image de-noising methods. 


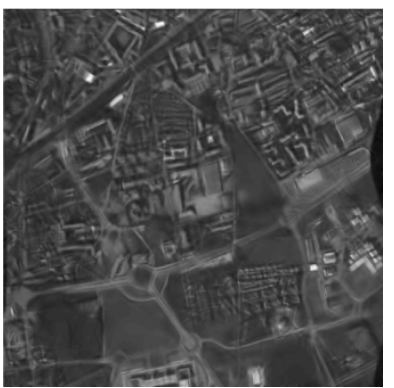

(a)

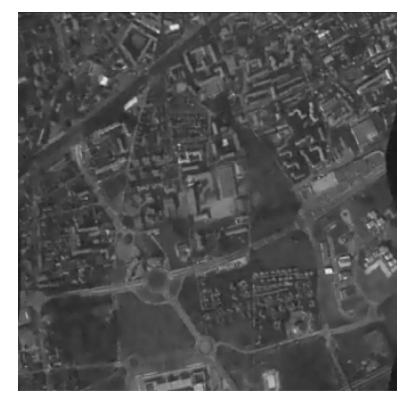

(d)

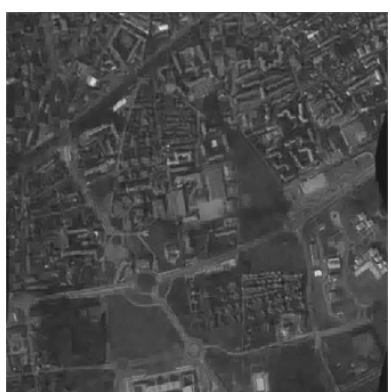

(b)

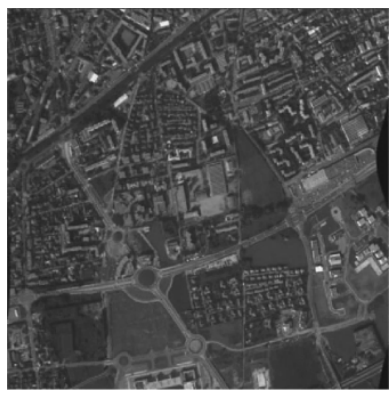

(e)

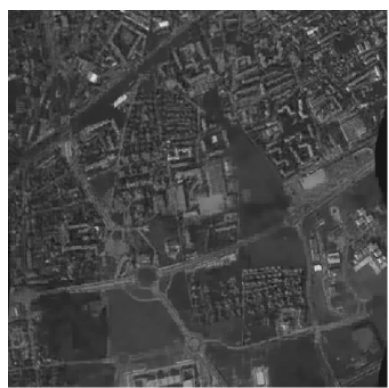

(c)

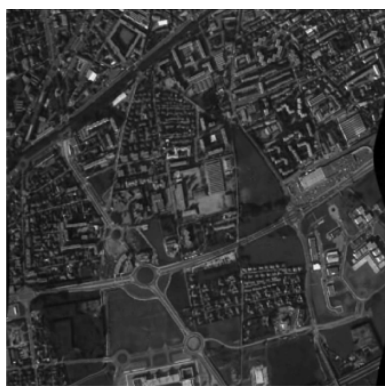

(f)

Fig. 5. The de-noised image of Fig. 4(b) (a) ST; (b) PPB; (c) SAR-BM3D; (d) K-SVD; (e) K-SVD+Wavelet; (f) The proposed

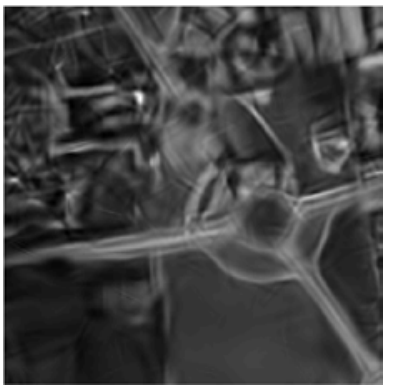

(a)

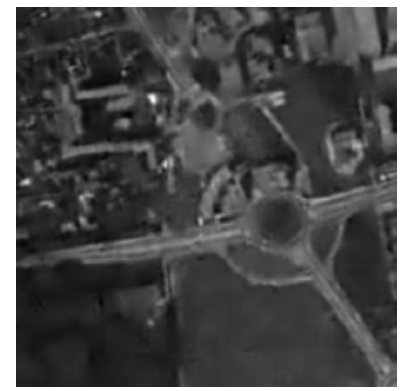

(d)

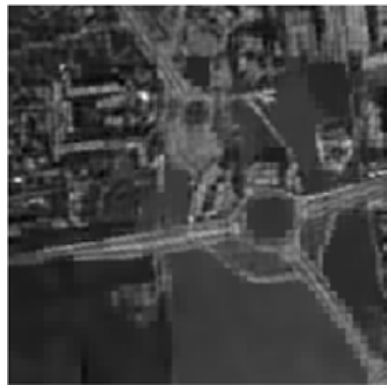

(b)

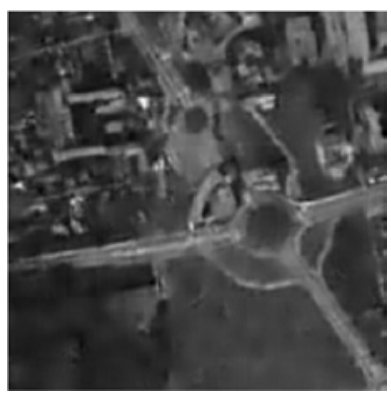

(e)

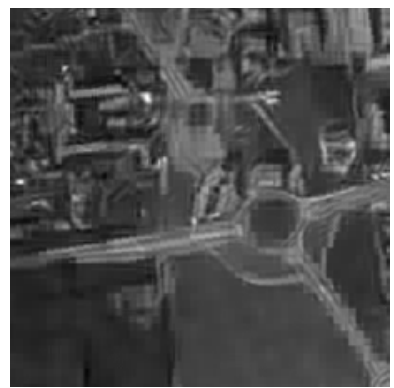

(c)

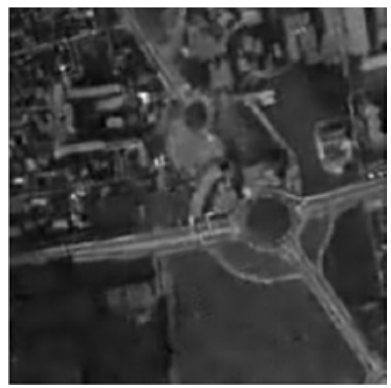

(f)

Fig. 6. The relevant de-noised region of Fig.4(b) (a) ST; (b) PPB; (c) SAR-BM3D; (d) K-SVD; (e) K-SVD+Wavelet; (f) The proposed

To testify the superiority of the proposed method objectively, we adopt some common objective evaluation indexes [18] to evaluate the de-noised images. The indexes include the 
peak signal to noise ratio (PSNR), equivalent number of looks (ENL), structural similarity (SSIM) and edge-preserving index (EPI). In the area of the image de-noising, we usually adopt PSNR to measure the ability of de-noising methods, and the larger the value is, the stronger the method is. ENL reflects the ability of distinguishing the different backward scattering areas, and the larger the value is, the better the visual effect is, and the weaker the speckle is. Moreover, SSIM stands for the similarity between the de-noised image and the original image, and the value of it varies from 0 to 1 . When the value of SSIM is larger, more information of the original image can be saved in the de-noised image, and more completed the structure of the de-noised image is. At last, EPI can reflect the ability of different methods to save the edges of the original, and the larger the value is, the better the ability is. When the value of EPI is larger than 1, the edges of the de-noised image is enhanced; otherwise, it is weakened.

When we add the speckle noise with $L=5 \sim 40$ to Fig. 4(a), the indexes of de-noised images by the aforementioned methods can be seen in Table 1. To exhibit the contents of Table 1 more intuitively, we change these indexes into the line chart shown in Fig. 7 where the color of all the indexes of the proposed method is black. From Table 1 and Fig. 7(a) we can see, compared with other methods, the de-noised images by the proposed method have improved a lot especially for the images with low speckle and had the highest PSNR. Meanwhile, the de-noised images by the proposed method have the highest ENL among the de-noised images of the same noisy image. All of these fully explain that the proposed method has better ability of image de-noising. Comparing the de-noised images of the same level noise in Table 1, we can obtain that SSIM of the de-noised image by the proposed method is the largest and all of them are over 0.95. As shown in Fig. 7(d), EPI of the de-noised image by the proposed method is best among all. Of all the image de-noising methods based on SR, because both of K-SVD+Wavelet and the proposed method make use of the residual images, EPI of de-noised images by them is larger than the one by K-SVD. But EPI of the proposed method is more close to 1 than K-SVD+Wavelet, which means the proposed has the best ability of saving the edges of the original image. What's more, these indexes strongly illustrate that the proposed method can save more information of the original image, especially for the edges and other details, further realizing the integrality of the image information.

Table 1. The indexes of the de-noised images of Fig. 4(a)

\begin{tabular}{|c|ccccc|}
\hline L/PSNR & The methods & PSNR & ENL & SSIM & EPI \\
\hline \multirow{5}{*}{$5 / 34.1515$} & ST & 23.3617 & 4.2211 & 0.9133 & 0.8555 \\
& PPB & 26.5198 & 4.2990 & 0.9223 & 0.8682 \\
& SAR-BM3D & 34.7567 & 4.3481 & 0.9872 & 0.8856 \\
& K-SVD & 36.8337 & 4.5041 & 0.9722 & 0.9279 \\
& K-SVD+Wavelet & 38.8751 & 5.1540 & 0.9870 & $\mathbf{1 . 0 1 1 0}$ \\
& The proposed & $\mathbf{5 0 . 2 2 0 4}$ & $\mathbf{5 . 5 7 6 9}$ & $\mathbf{0 . 9 8 8 9}$ & 1.0114 \\
\hline \multirow{5}{*}{$10 / 28.1381$} & ST & 26.9254 & 4.1683 & 0.8875 & 0.8102 \\
& PPB & 28.1407 & 4.3901 & 0.8982 & 0.8457 \\
& SAR-BM3D & 30.8434 & 4.5682 & 0.9144 & 0.8232 \\
& K-SVD & 32.3496 & 4.8286 & 0.9776 & 0.8759 \\
& K-SVD+Wavelet & 34.8589 & 5.5322 & 0.9800 & $\mathbf{1 . 0 6 0 8}$ \\
& The proposed & $\mathbf{4 5 . 6 6 7 6}$ & $\mathbf{6 . 0 0 1 6}$ & $\mathbf{0 . 9 8 8 4}$ & 1.1941 \\
\hline
\end{tabular}




\begin{tabular}{|c|c|c|c|c|c|}
\hline \multirow{6}{*}{$15 / 24.6170$} & ST & 24.3883 & 4.0936 & 0.9011 & 0.7001 \\
\hline & РРВ & 26.9442 & 4.4802 & 0.9159 & 0.7177 \\
\hline & SAR-BM3D & 28.8670 & 4.8068 & 0.9471 & 0.7891 \\
\hline & K-SVD & 30.0414 & 5.1628 & 0.9410 & 0.8008 \\
\hline & K-SVD+Wavelet & 32.9294 & 5.3723 & 0.9604 & 0.8959 \\
\hline & The proposed & 43.1711 & 5.8347 & 0.9858 & 0.9316 \\
\hline \multirow{6}{*}{ 20/22.1175 } & ST & 26.4213 & 4.0424 & 0.9038 & 0.6623 \\
\hline & РPB & 26.8824 & 4.5570 & 0.9166 & 0.6740 \\
\hline & SAR-BM3D & 27.5226 & 5.1094 & 0.9211 & 0.7248 \\
\hline & K-SVD & 28.4941 & 5.1023 & 0.9259 & 0.7527 \\
\hline & K-SVD+Wavelet & 31.7358 & 5.5003 & 0.9427 & 0.8708 \\
\hline & The proposed & 41.5195 & 5.5642 & 0.9685 & 0.9766 \\
\hline \multirow{6}{*}{$25 / 20.1694$} & ST & 26.0563 & 4.3705 & 0.8989 & 0.7029 \\
\hline & РPB & 26.4654 & 4.6148 & 0.9043 & 0.8989 \\
\hline & SAR-BM3D & 26.5256 & 4.7114 & 0.9116 & 0.8316 \\
\hline & K-SVD & 27.2401 & 5.1555 & 0.9214 & 0.8795 \\
\hline & K-SVD+Wavelet & 30.8866 & 5.4261 & 0.9393 & 0.9116 \\
\hline & The proposed & 35.0317 & 5.8537 & 0.9536 & 1.0117 \\
\hline \multirow[t]{6}{*}{ 30/18.6070 } & ST & 24.4678 & 3.9334 & 0.8650 & 0.4558 \\
\hline & РРВ & 24.6517 & 4.3653 & 0.8771 & 0.4755 \\
\hline & SAR-BM3D & 25.7291 & 4.6565 & 0.8825 & 0.5067 \\
\hline & K-SVD & 26.2721 & 4.8061 & 0.8991 & 0.6392 \\
\hline & K-SVD+Wavelet & 30.1838 & 5.7887 & 0.9353 & 0.8481 \\
\hline & The proposed & 30.8969 & 6.1980 & 0.9557 & 1.0367 \\
\hline \multirow{6}{*}{ 35/17.2477 } & ST & 20.5779 & 2.7071 & 0.7459 & 0.6207 \\
\hline & РPB & 21.9530 & 3.4057 & 0.7887 & 0.6718 \\
\hline & SAR-BM3D & 25.1329 & 4.6898 & 0.8623 & 0.6726 \\
\hline & K-SVD & 25.4647 & 5.2509 & 0.8751 & 0.7215 \\
\hline & K-SVD+Wavelet & 28.6523 & 6.2201 & 0.9182 & 0.9552 \\
\hline & The proposed & 28.9688 & 6.5368 & 0.9641 & 1.0215 \\
\hline \multirow{6}{*}{ 40/16.1008 } & ST & 19.6012 & 1.8513 & 0.6012 & 0.4815 \\
\hline & РPB & 17.5562 & 2.5711 & 0.6913 & 0.6186 \\
\hline & SAR-BM3D & 24.6513 & 4.7286 & 0.8446 & 0.6357 \\
\hline & K-SVD & 24.8053 & 5.7101 & 0.8512 & 0.6335 \\
\hline & K-SVD+Wavelet & 27.1647 & 6.6029 & 0.8981 & 0.7355 \\
\hline & $\mathrm{Tl}$ & 27.6607 & 6.7092 & 0.9700 & 0.9146 \\
\hline
\end{tabular}




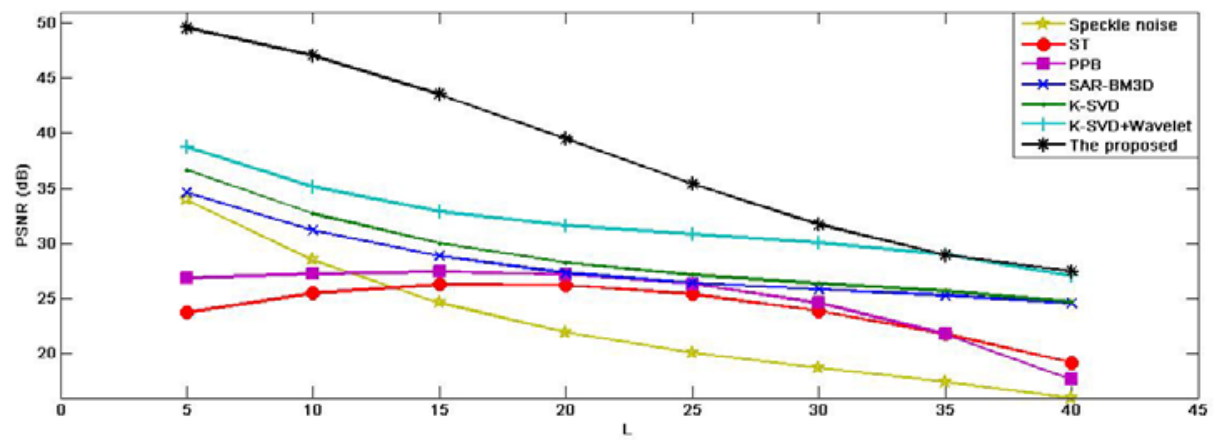

(a)

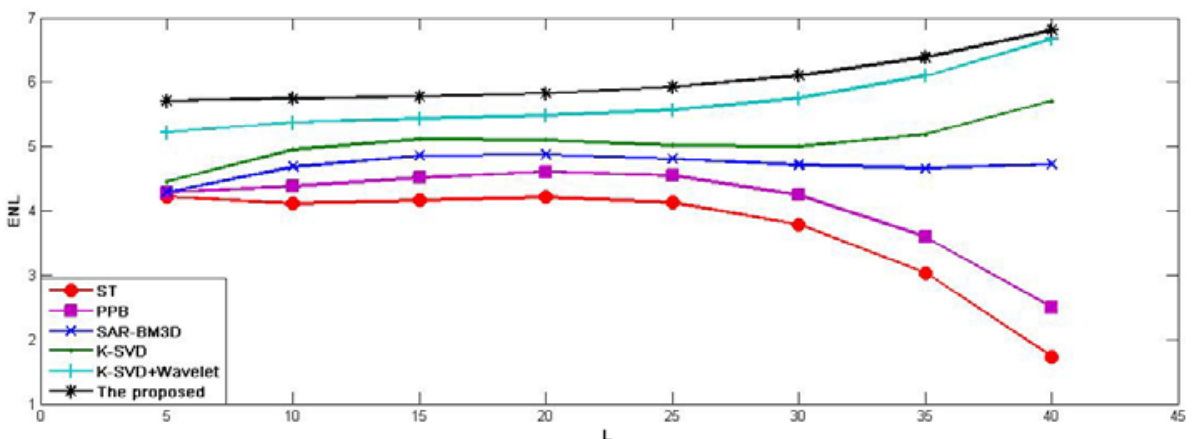

(b)

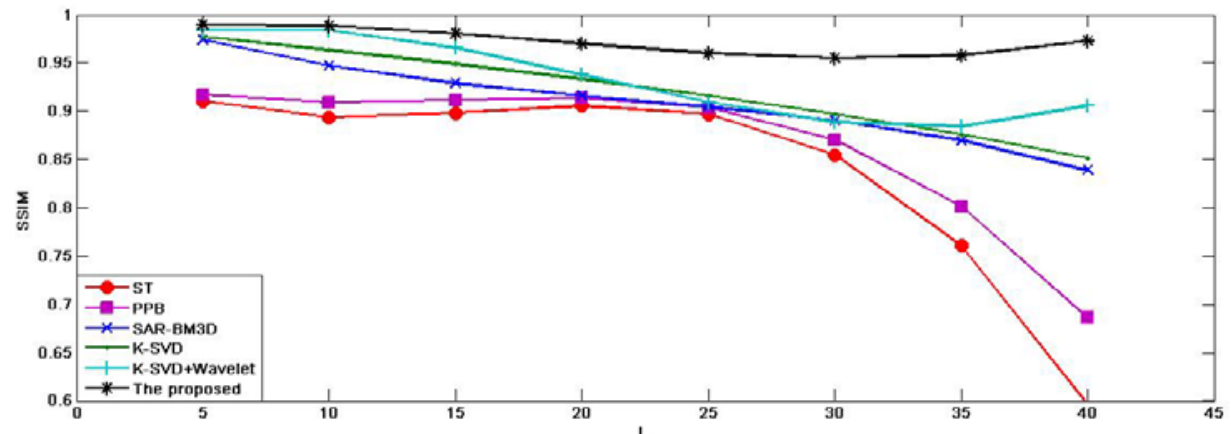

(c)

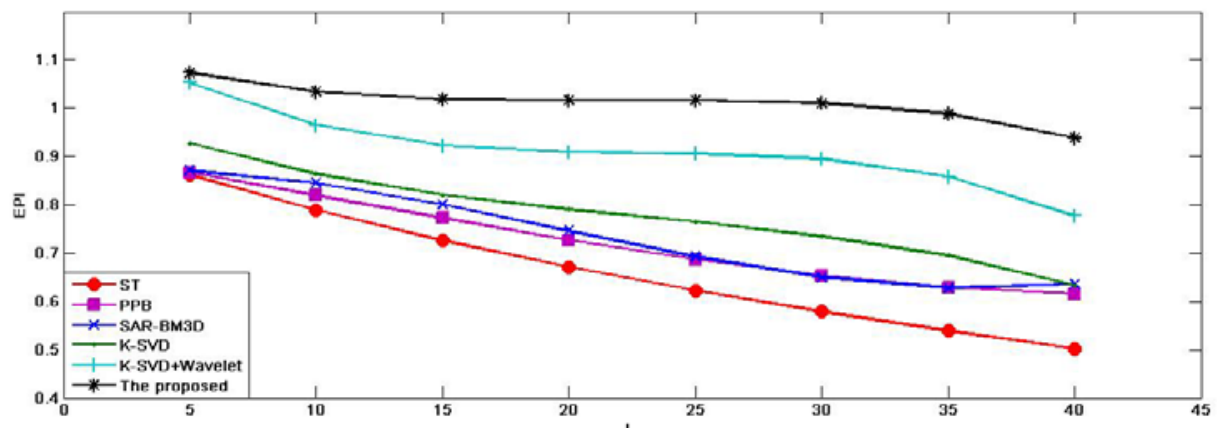

(d)

Fig. 7. The line chart of Table 1 (a) PSNR; (b) ENL; (c) SSIM; (d) EPI 
To more fully account for the universality and practicability of the proposed method, next we perform the experiments with the SAR images of which the size is $512 \times 512$ and $256 \times 256$, and analysis their result carefully. Fig. 8(a) and Fig. 9(a) are the original SAR images; and we add the noise with $L=25$ to them. Then we can obtain the corresponding noisy images shown as Fig. 8(b) and Fig. 9(b).

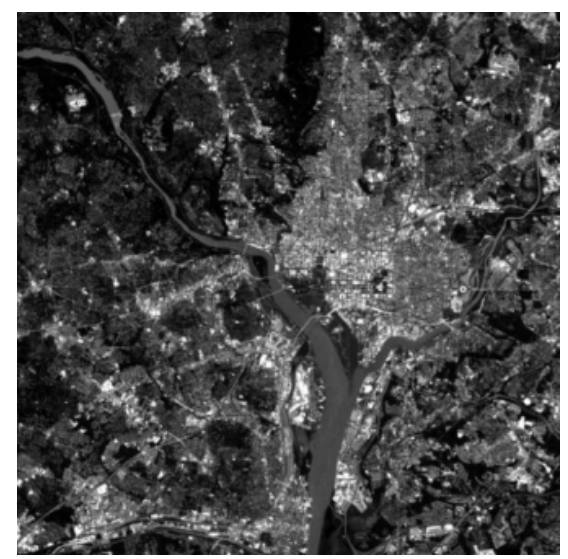

(a)

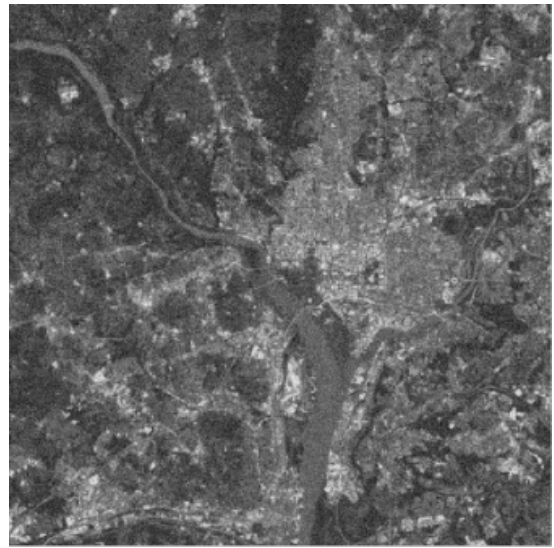

(b)

Fig. 8. The SAR images with size of $512 \times 512$ (a) the original image; (b) the noisy image

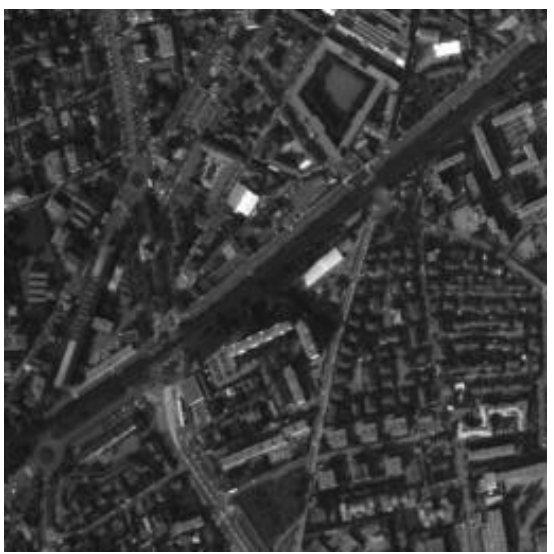

(a)

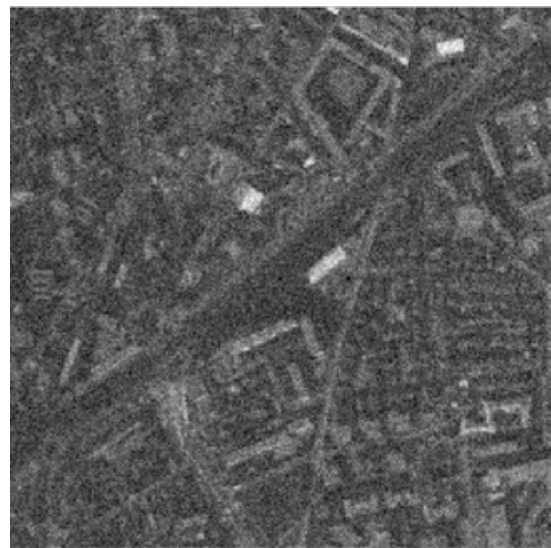

(b)

Fig. 9. The SAR images with size of $256 \times 256$ (a) the original image; (b) the noisy image

Similarly, we de-noise the noisy image Fig. 8(b) by the aforementioned methods and can obtain the de-noised images shown in Fig. 10. Besides, as shown in Table 2, the aforementioned indexes are adopted to evaluate the de-noised images in Fig. 10. As shown in Fig. 10(a), the de-noised image by ST is very blurry, especially for the edges which are difficult to distinguish. Due to the existence of the artificial texture in Fig. 10(b) and (c), there is a mixture of the artificial texture and the edges, which leads to serious influence on the subsequent image processing; there is still some noise in the two de-noised images as well. From Fig. 10(d) we can see, the de-noised image by K-SVD has less noise, but it loses a lot of important information during the processing of the image de-noising. For example, the 
reflection of the bridge in the wide river almost cannot be recognized. Compared with the other de-noised images, Fig. 10(e) and (f) not only have less noise, but also save more detail information of the original image. However, Fig. 10(f) has the best visual effect which shows that the proposed method is the best of all.

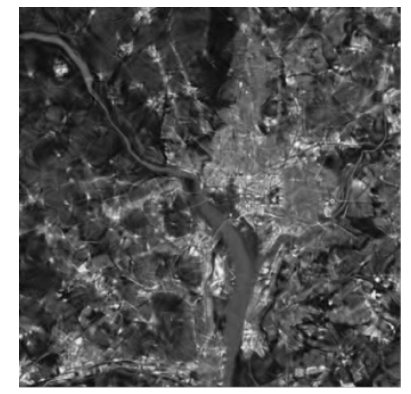

(a)

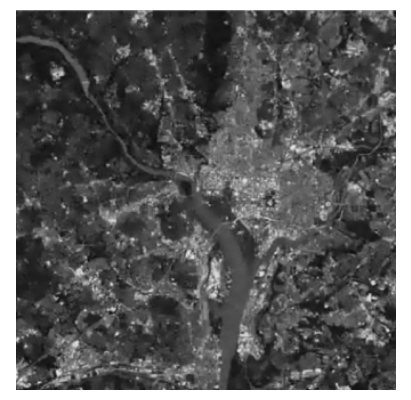

(d)

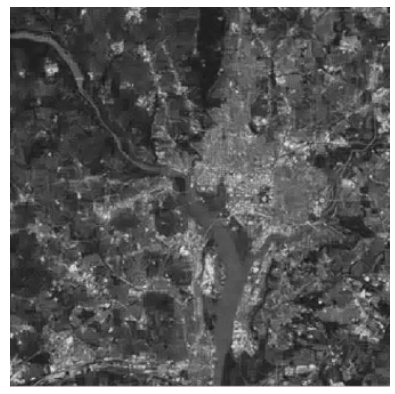

(b)

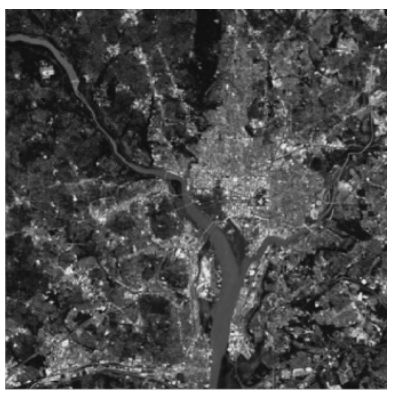

(e)

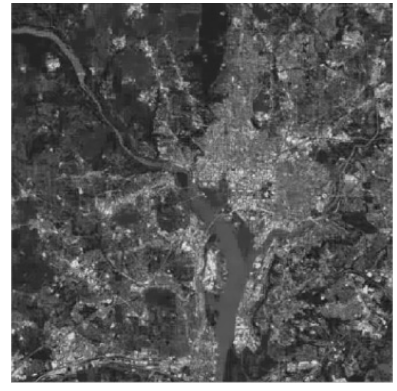

(c)

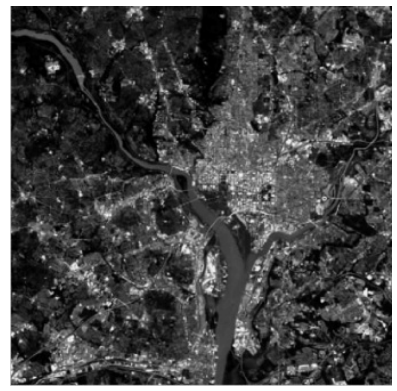

(f)

Fig. 10. The de-noised image of Fig. 8(b) (a) ST; (b) PPB; (c) SAR-BM3D; (d) K-SVD; (e) K-SVD+Wavelet; (f) The proposed

Table 2. The indexes of the de-noised images in Fig. 10

\begin{tabular}{ccccc}
\hline The methods & PSNR & ENL & SSIM & EPI \\
\hline ST & 24.8843 & 2.0348 & 0.9472 & 0.3817 \\
PPB & 24.5590 & 2.0735 & 0.9452 & 0.4797 \\
SAR-BM3D & 24.9549 & 2.0774 & 0.9516 & 0.6881 \\
K-SVD & 25.9178 & 2.1754 & 0.9594 & 0.6607 \\
K-SVD+Wavelet & 29.2150 & 2.2478 & 0.9776 & 0.7750 \\
The proposed & 33.3009 & 2.3774 & 0.9793 & 0.9104 \\
\hline
\end{tabular}

By comparing the indexes in Table 2, we can easily come to the conclusion that the proposed method can improve PSNR of the de-noised image and realize better image de-noising. What's more, ENL of the de-noised image by the proposed method is also the best; and SSIM is close to 1. EPI of the de-noised image by the proposed method is the highest and over 0.9. All of these illustrate that the proposed method is optimal among the aforementioned methods. 
Fig. 11 and Table 3 are the results of de-noised images of Fig. 9(b) by different image de-noising methods and the corresponding objective evaluation indexes of them respectively. From Fig. 11 we can see, the proposed method not only suppresses the speckle noise effectively, but also saves more detail information and has the best visual effect. On the other hand, Table 3 shows that the indexes of the proposed method are the best, and PSNR and ENL have improved a lot. Moreover, SSIM and EPI are closer to 1. Therefore, the proposed method is effective for the image de-noising.

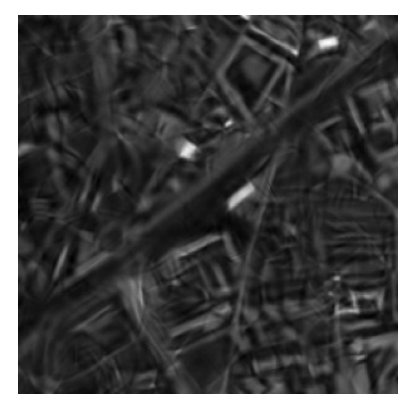

(a)

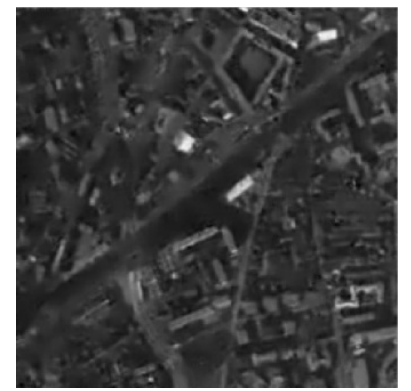

(d)

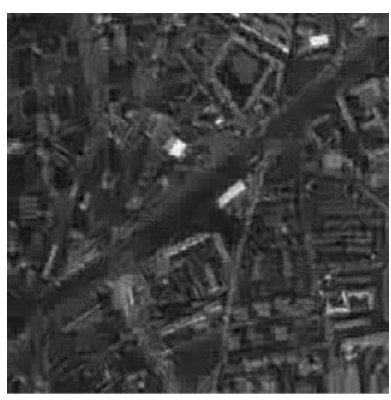

(b)

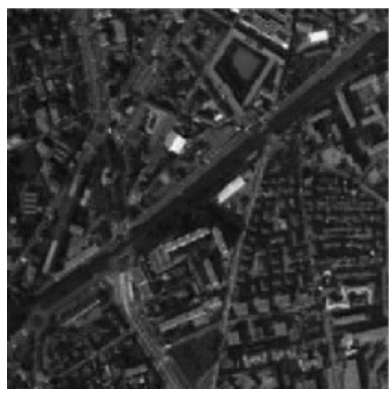

(e)

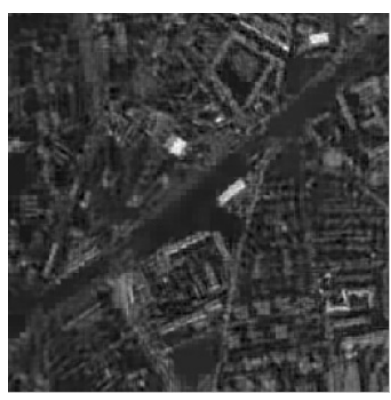

(c)

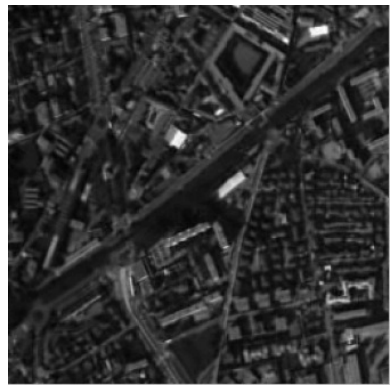

(f)

Fig. 11. The de-noised image of Fig. 9(b) (a) ST; (b) PPB; (c) SAR-BM3D; (d) K-SVD; (e) K-SVD+Wavelet; (f) The proposed

Table 3. The indexes of the de-noised images in Fig. 11

\begin{tabular}{ccccc} 
The methods & PSNR & ENL & SSIM & EPI \\
\hline ST & 26.3351 & 4.4735 & 0.8940 & 0.5762 \\
PPB & 25.6846 & 4.5084 & 0.8829 & 0.6566 \\
SAR-BM3D & 26.1674 & 4.6410 & 0.8999 & 0.7692 \\
K-SVD & 26.8846 & 5.1580 & 0.9091 & 0.7537 \\
K-SVD+Wavelet & 30.7469 & 5.4155 & 0.9440 & 0.8866 \\
The proposed & 33.8779 & 5.8195 & 0.9598 & 0.9361 \\
\hline
\end{tabular}

\section{Conclusion}

By combining the merits of the image de-noising method based on sparse representation and making full use of the detail information in the residual image, in this paper, a novel SAR 
image de-noising method based on sparse representation and the residual image fusion in Shearlet domain is proposed. Experimental results show that the proposed method has improved a lot on the subjective visual effects and objective evaluation indexes, and it is an efficient and practical SAR image de-noising method. However, in the proposed method we cascade some complementary SAR image de-noising methods and the image de-noising method based on SR is time-consuming, the time complexity is high. In our future work, we will try to improve it.

\section{Future Scope}

As one of effective means for observing from the space to earth, SAR imaging does not rely on the illumination but has its microwave. It can penetrate most obstacles such as the cloud, rain and the material on the surface. Especially for military applications and environmental testing, SAR image has big significance. However, one single point in SAR image may correspond to one building in reality. In some cases, it is hard to distinguish the speckle noise and the detail information in SAR image. As a result, the speckle noise of SAR image makes the image information incorrect and can mislead us sometime. Effectively suppressing or removing the noise by the image de-noising methods make the SAR image more useful and is very important for the subsequent image processing.

\section{References}

[1] Hu Shaohai, Ma Xiaole, Liu Shuaiqi, and Yang Dongsheng, "SAR image de-noising based on non-local similar block matching in NSST domain,” in Proc. of 2016 IEEE $13^{\text {th }}$ International Conference on Signal Processing, pp.832-836, November 6-10, 2016. Article (CrossRef Link).

[2] Ling Shao, Ruomei Yan, Xuelong Li, and Yan Liu, "From heuristic optimization to dictionary learning: a review and comprehensive comparison of image denoising algorithms," IEEE Transactions on Cybernetics, vol. 44, no. 7, pp. 1001-1013, July, 2014. Article (CrossRef Link).

[3] Michal Aharon, Michael Elad and Alfred Bruckstein, “The K-SVD : an algorithm for designing of overcomplete dictionaries for sparse representation,” IEEE Transactions on Signal Processing, vol. 54, no. 11, pp. 4311-4322, November, 2006. Article (CrossRef Link).

[4] Shi Guangming, Liu Danhua, Gao Dahua and et.al., "Advances in Theory and Application of Compressed Sensing,” Acta Electronica Sinica, vol. 37, no. 5, pp. 1070-1081, May, 2009. Article (CrossRef Link).

[5] Chenglong Wang, Guanghui Zhao, Guangming Shi and Huan Li, "SAR image despeckling based on the nonlocally centralized sparse representation model," in Proc. of 2014 IEEE International Conference on Computer and Information Technology, pp. 143-147, September 11-13, 2014. Article (CrossRef Link).

[6] Xiao Quan, Ding Xinghao, Wang Shoujue and et.al., "Image denoising based on adaptive over-complete sparse representation,” Chinese Journal of Scientific Instrument, vol. 30, no. 9, pp. 1886-1890, September, 2009. Article (CrossRef Link).

[7] Michael Elad and Michal Aharon, "Image denoising via sparse and redundant representation over learned dictionaries,” IEEE Transactions on Signal Processing, vol. 15, no. 12, pp. 3736-3745, December, 2006. Article (CrossRef Link).

[8] Bhabesh Deka and Prabin Kumar Bora, "Removal of correlated speckle noise using sparse and overcomplete representations,” Biomedical Signal Processing and Control, vol. 8, no. 6, pp. 520-533, November, 2013. Article (CrossRef Link). 
[9] A. Buades, B. Coll and J.M. Morel, "A review of image denoising algorithms, with a new one," Multiscale Model. Simul., vol. 4, no. 2, pp. 490-530, 2005. Article (CrossRef Link).

[10] Hu Shaohai, Yang Dongsheng, Liu Shuaiqi, and Xiaole Ma, "Block-matching based mutimodal medical image fusion via PCNN with SML," in Proc. of 2016 IEEE $13^{\text {th }}$ International Conference on Signal Processing, pp. 13-18, November 6-10, 2016. Article (CrossRef Link).

[11] Kanghui Guo and Demetrio Labate, "Optimally sparse multidimensional representation using Shearlets,” SIAM Journal on Mathematical Analysis, vol. 39, no. 1, pp. 298-318, 2007. Article (CrossRef Link).

[12] Donoho D L, "De-noising by soft-thresholding," IEEE transactions on information theory, vol. 41, no. 3, pp. 613-627, May, 1995. Article (CrossRef Link).

[13] Dongming Li, Lijuan Zhang, Jinhua Yang and Wei Su, "Research on wavelet-based contourlet transform algorithm for adaptive optics image denoising," Optik-International Journal for Light and Electron Optics, vol. 127, no. 12, pp. 5029-5034, June, 2016. Article (CrossRef Link).

[14] Goodman J W, "Some fundamental properties of speckle," Journal of the optical society of America, vol. 66, no. 11, pp. 1145-1150, November, 1976. Article (CrossRef Link).

[15] Charles-Alban Deledalle, LoIc Denis and Florence Tupin, "Iterative weighted maximum likelihood denoising with probabilistic patch-based weights," IEEE Trans. Image Process., vol. 18, no. 12, pp. 2661-2672, December, 2009. Article (CrossRef Link).

[16] Sara Parrilli, Mariana Poderico, Cesario Vincenzo Angelino and Luisa Verdoliva, "A nonlocal SAR image denoising algorithm based on LLMMSE wavelet shrinkage," IEEE Trans. Geosci. Remote Sens., vol. 50, no. 2, pp. 606-616, February, 2012. Article (CrossRef Link).

[17] Dong Mingkun, Jiang Aimin and Sun Juan, "Dictionary-learning-based image denoising method using information fusion of residuals,” Microprocessors, vol. 2, no. 1, pp. 58-63, 2015.

Article (CrossRef Link).

[18] Liu Shuaiqi, Hu Shaohai, and Xiao Yang, "Bayesian shearlet shrinkage for SAR image de-noising via sparse representation,” Multidimensional Systems and Signal Processing, vol. 25, no. 4, pp. 683-701, October, 2014. Article (CrossRef Link). 


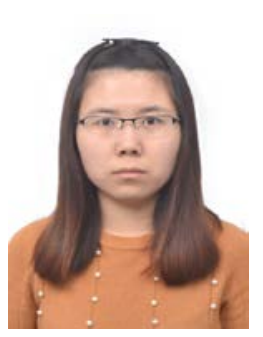

Xiaole Ma was born in 1991. She received her B.S. degree in Communication Engineering at Electronic Information Engineering, Hebei University. At present, she is pursuing her Ph.D. degree in Signal and Information Processing at the Institute of Information Science, Beijing Jiaotong University. Currently, her research interests include image de-noising, image fusion and signal processing.

E-mail: maxiaole@bjtu.edu.cn

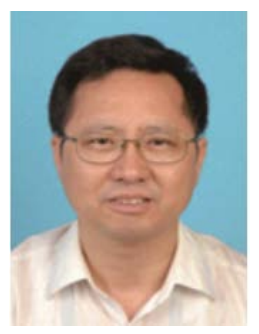

Shaohai Hu was born in 1964. He received his Ph.D. degrees in Institute of Information Science from Beijing Jiaotong University in 1991 and has been a professor of Institute of Information Science since 2008. His research interests lie in the broad area of signal processing and information fusion, include image fusion, image denosing and sparse representation.

E-mail: shhu@bjtu.edu.cn

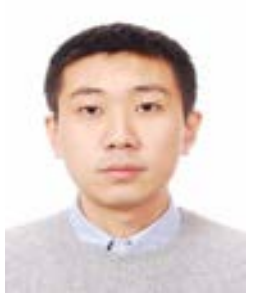

Dongsheng Yang was born in 1991. He is pursuing his M.Sc. degree in Information and Signal Processing at the Institute of Information Science, Beijing Jiaotong University. Currently, his research interests include image fusion, image denoising and signal processing.

E-mail: dsyang@bjtu.edu.cn 